of rectal bleeding. Endoscopically reported findings of inflammation were collated and the pathological findings at biopsy reviewed. Patients with prediagnosed IBD were excluded from the analysis.

Results 1391 procedures were carried out on 1240 individuals. Endoscopically reported inflammation was seen in 54 (4.4\%) and pathologically confirmed IBD found in 35 (2.8\%). Of these 16 were diagnosed as having Crohn's disease (1.3\%) and 19 ulcerative colitis $(1.5 \%)$. The other final diagnoses are shown in table 1 below. Of the 10 patients determined to have indeterminate colitis at histology, a further $7(0.4 \%)$ had pathological findings of IBD but lacked diagnostic features.

Of those with confirmed IBD, 10 with Crohn's disease (62\%) and 9 with ulcerative colitis (47\%) had not reported gastrointestinal symptoms.

Conclusion In this cohort of 1240 patients, a confirmed new diagnosis of inflammatory bowel disease was found in $2.8 \%$. A further $0.4 \%$ had histological changes that were likely to represent IBD. Of those with a diagnosis of IBD, 54\% were asymptomatic on direct questioning prior to the procedure. Previously undiagnosed IBD is a frequent finding at screening colonoscopy even in patients without symptoms.

Competing interests None.

Keywords Crohn's disease, screening, ulcerative.

\section{PTH-057 NEW DIAGNOSES OF INFLAMMATORY BOWEL DISEASE DURING BOWEL CANCER SCREENING COLONOSCOPY}

doi:10.1136/gut.2011.239301.458

J Louis-Auguste, 1, ${ }^{1}$ P Cohen, ${ }^{2} \mathrm{~J}$ Martin, ${ }^{1} \mathrm{G}$ V Smith ${ }^{1}$ Department of Gastroenterology, Imperial College Healthcare NHS Trust, London, UK; ${ }^{2}$ Department of Pathology, Imperial College Healthcare NHS Trust, London, UK

Introduction Colonoscopy in the National Bowel Cancer Screening Programme is designed to detect neoplasia based on positive faecal occult blood testing in an asymptomatic population between the ages of 60 and 75; however participants often report symptoms at the time of assessment. Anecdotally some individuals have been reported to have inflammatory bowel disease at screening. The aim of this study is to quantify the number of histologically confirmed new diagnoses of IBD in this population and the proportion of these that have symptoms.

Methods The authors retrospectively identified all patients who underwent screening colonoscopy during the first 3 years of the programme at Charing Cross Hospital (Screening population 1.1 million). Gastrointestinal symptoms were prospectively recorded at the initial interview. Specifically individuals were asked about bowel frequency, diarrhoea or the presence

Table 1 PTH-057

\begin{tabular}{llclll}
\hline Endoscopic appearance & Pathology UC & Pathology Crohn's & Pathology diverticular & Pathology ischaemic & Pathology other \\
\hline UC 22 & 18 & 1 & & 1 & 2 \\
Crohn's 13 & 12 & 3 & 2 & 3 & 10 \\
Indeterminate 19 & 1 & 3 & 1 & \\
\hline
\end{tabular}

